\title{
Estimativa da produtividade de café com base em um modelo agrometeorológico-espectral
}

\author{
Viviane Gomes Cardoso da Rosa(1), Maurício Alves Moreira(1), \\ Bernardo Friedrich Theodor Rudorff( ${ }^{(1)}$ e Marcos Adami ${ }^{(1)}$
}

(1)Instituto Nacional de Pesquisas Espaciais, Divisão de Sensoriamento Remoto, Avenida dos Astronautas, no 1.758, CEP 12227-010 São José dos Campos, SP. E-mail: vivian@dsr.inpe.br, mauricio@dsr.inpe.br, rudorff@dsr.inpe.br, adami@dsr.inpe.br

\begin{abstract}
Resumo-Oobjetivo deste trabalho foi avaliarum modelo agrometeorológico-espectral, para estimar a produtividade de cafezais. Utilizaram-se imagens do sensor MODIS e dados agrometeorológicos do modelo regional de previsão do tempo (ETA), para fornecer as variáveis de entrada para o modelo agrometeorológico-espectral da mesorregião geográfica sul/sudoeste do estado de Minas Gerais nos anos-agrícolas de 2003/2004 a 2007/2008. A variável espectral de entrada do modelo agrometeorológico-espectral, índice de área foliar (IAF), usada no cálculo da produtividade máxima, foi estimada com o índice de vegetação por diferença normalizada (NDVI), obtido de imagens MODIS. Outras variáveis de entrada no modelo foram: dados meteorológicos gerados pelo modelo ETA e a capacidade de água disponível no solo. Ao comparar a produtividade média estimada pelo modelo com a fornecida oficialmente pelo IBGE, as diferenças relativas obtidas em escala regional foram de: $0,4,3,0,5,3$, 1,5 e 8,5\% para os anos agrícolas 2003/2004, 2004/2005, 2005/2006, 2006/2007 e 2007/2008, respectivamente. O modelo agrometeorólogico-espectral, que tem como base o modelo de Doorenbos \& Kassan, foi tão eficaz para estimar a produtividade dos cafezais quanto o modelo oficial do IBGE. Além disso, foi possível espacializar a quebra de produtividade e prever $80 \%$ da produtividade final na primeira quinzena de fevereiro, antes do início da colheita.
\end{abstract}

Termos para indexação: Coffea, estatísticas agrícolas, índice de área foliar, modelagem, sensoriamento remoto.

\section{Coffee crop yield estimate using an agrometeorological-spectral model}

\begin{abstract}
The objective of this work was to evaluate an agrometeorological-spectral model to estimate coffee crop yield. Images from the MODIS sensor and meteorological data from the ETA regional weather forecast model were used to provide input variables to the agrometeorological-spectral model, in the South-Southeast region of Minas Gerais State, Brazil, for crop years 2003/2004 to 2007/2008. The input spectral variable of the spectral-agrometeorological model, the leaf area index (LAI), used in the determination of the maximum yield, was estimated with the normalized-difference vegetation index (NDVI) obtained from MODIS images. Other input variables for the model were: meteorological data generated by the ETA model and the soil available water capacity. Comparing the estimated model average crop yield with those from IBGE, it was verified that the relative differences, at regional scale, were: $0.4 ; 3.0 ; 5.3 ; 1.5$ and $8.5 \%$ for crop years $2003 / 2004$, 2004/2005, 2005/2006, 2006/2007 and 2007/2008, respectively. The agrometeorological-spectral model, based on Doorenbos \& Kassan model, was as efficient as the IBGE official model to estimate the coffee crop yield. Furthermore, it was possible to present the spatial variation of coffee crop yield loss and to predict $80 \%$ of final yield by the first fortight of February before the harvest.
\end{abstract}

Index terms: Coffea, agricultural statistics, leaf area index, modeling, remote sensing.

\section{Introdução}

No Brasil, a quantificação dos efeitos da variabilidade climática em culturas agrícolas e, consequentemente, o impacto sobre a produção são realizados de duas maneiras. Na primeira, inteiramente subjetiva, a safra é estimada com informações baseadas em opiniões de agentes técnicos e econômicos do setor agrícola.
A informação gerada a partir dessa abordagem não permite uma análise apurada dos erros envolvidos. Pino (2001) comenta ainda que, devido à grande extensão territorial, essas informações nem sempre são atualizadas.

$\mathrm{Na}$ segunda, a safra é estimada em razão da quebra de rendimento com base em modelos agrometeorológicos e agrometeorológicos-espectrais, quando se utilizam informações multiespectrais de

Pesq. agropec. bras., Brasília, v.45, n.12, p.1478-1488, dez. 2010 
imagens de satélites para calcular algumas variáveis intrínsecas ao modelo.

Os modelos agrometeorológicos foram os primeiros a serem desenvolvidos e são muito utilizados atualmente. Além de fornecer dados para alimentar os sistemas de previsão de safras agrícolas, permitem identificar estresse hídrico ao longo do ciclo da cultura, cujos impactos na produtividade podem ser detectados e avaliados (Doorenbos \& Kassam, 1979; Rudorff \& Batista, 1990; Camargo et al., 1999).

Sobre a cultura do café no Brasil, são encontradas pesquisas com enfoque na estimativa da produtividade em Tosello \& Arruda (1962), Silva et al. (1987), Liu \& Liu (1988), Weill (1990), Picini et al. (1999), Carvalho et al. (2003), Santos \& Camargo (2006). Todavia, a parametrização de um modelo agrometeorológico para a cultura do café não é tarefa fácil, pois existem diversos fatores que influenciam a produtividade do café, tais como: variedade cultivada, sistema de plantio (adensado e não adensado), idade da lavoura, tipo e fertilidade do solo e sistema de manejo. Além disso, a característica de bienalidade de produção do cafeeiro e a falta de um mapa temático com a distribuição espacial da área cultivada aumentam ainda mais a complexidade da estimativa da produtividade do café em grandes áreas territoriais por meio desses modelos. Picini (1999) parametrizou e testou diversos modelos agrometeorológicos pontuais e, entre eles, o modelo proposto por Doorenbos \& Kassam (1979) foi o que permitiu obter melhor resultado após a adaptação da produtividade do ano anterior.

Segundo Rudorff \& Batista (1990), parte dessas questões pode ser solucionada ao serem incorporadas, no modelo, informações espectrais de culturas agrícolas, obtidas em imagens de satélites de sensoriamento remoto, expressas como índice de vegetação.

Apesar da comprovada utilidade dos índices de vegetação, obtidos de imagens de satélites de média resolução espacial, como as do TM/Landsat-5, do CCD/CEBRS-2B, HRV/SPOT, essas imagens têm sido pouco utilizadas para estimar a produtividade de culturas agrícolas devido à baixa resolução temporal - pouca disponibilidade de imagens - e à alta probabilidade de incidência de nuvens durante o período de crescimento e desenvolvimento das culturas. Com o advento do sensor MODIS (Moderate Resolution Imaging Spectroradiometer), a bordo dos satélites Terra e Aqua, com resolução temporal diária, houve aumento significativo da disponibilidade de imagens livres de nuvens. Além disso, a cada 16 dias, é colocada à disposição, para download, uma imagem livre de nuvens, proveniente de um mosaico dos melhores pixels observados nas 16 imagens diárias. Esse procedimento tem possibilitado o uso de imagens multiespectrais em modelos de estimativa de produtividade para monitorar o crescimento de culturas agrícolas em grandes áreas (Rizzi, 2004).

Em vista disso, a hipótese deste trabalho foi a de que imagens MODIS e dados meteorológicos, obtidos com previsões do modelo regional ETA, podem ser utilizados para estimar a produtividade média dos cafezais no âmbito regional.

O objetivo deste trabalho foi avaliar o potencial das imagens do sensor MODIS e dos dados do modelo regional ETA no modelo agrometeorológico-espectral para estimar a produtividade dos cafezais.

\section{Material e Métodos}

A área de estudo está localizada na mesorregião geográfica sul/sudoeste do estado de Minas Gerais, entre os paralelos $20^{\circ} 10^{\prime}$ e $23^{\circ} 00^{\prime} \mathrm{S}$ e os meridianos $43^{\circ} 59^{\prime}$ e $47^{\circ} 20^{\prime} \mathrm{W}$. Ela é composta por 144 municípios que abrangem uma área de

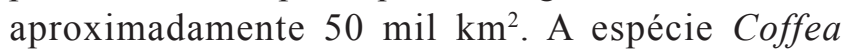
arabica é a predominante nessa região, responde por $46 \%$ de todo o café produzido no estado de Minas Gerais e representa aproximadamente 31\% da produção brasileira (Instituto Brasileiro de Geografia e Estatística, 2010).

A altitude média da região é de aproximadamente $950 \mathrm{~m}$, com relevo predominantemente ondulado a forte ondulado. O clima da região, segundo a classificação de Köppen, é o Cwb com estação chuvosa no verão e temperatura média mensal inferior a $18^{\circ} \mathrm{C}$. A temperatura mínima anual no inverno é de 5 a $6^{\circ} \mathrm{C}$. Nos meses mais quentes, a temperatura pode ultrapassar $34^{\circ} \mathrm{C}$. A pluviosidade média é de $1.623 \mathrm{~mm}$ por ano (Sediyama et al., 2001). As classes de solo predominantes na 
região são Latossolos Vermelho Escuro, Vermelho Amarelo e Latossolo Roxo; Argissolos Vermelho Amarelo; Cambissolos; Neossolo Litólico e Neossolo Quartzarênico (Geominas, 1996).

A cultura do café passa por seis fases fenológicas, duas vegetativas e quatro reprodutivas, num período de dois anos para completar o seu ciclo de produção (Camargo \& Camargo, 2001). Por causa dessa bienalidade da planta de café, a produção alterna-se entre anos de alta e baixa produ tividade.

O modelo agrometeorológico-espectral utilizado tem por base o modelo proposto por Doorenbos \& Kassam (1979), expresso segundo a equação 1:

Yest $=\mathrm{Yp}[1-\mathrm{ky}(1-\mathrm{ETr} / \mathrm{ETm})]$

em que: Yest é a produção real, Yp, a produção máxima, ky é o fator de resposta à produtividade, ETr é a evapotranspiração real e ETm, a evapotranspiração máxima. A produtividade máxima (Yp) é estimada pela equação 2:

Yp $=$ Fcc $\times$ Fr $\times$ Fpa $\times$ ND $\times$ PMB

em que: Fcc é o fator de compensação do crescimento, Fr, o fator de respiração da cultura, Fpa é o fator de produtividade agrícola, ND é o período de tempo entre duas estimativas consecutivas (em dias), PMB, a produção de massa de matéria seca bruta do grupo da cultura (Rudorff \& Batista, 1990).

O Fcc é calculado pela equação 3, que foi definida por Berka et al. (2003) com base em dados tabulares apresentados em Doorenbos \& Kassam (1979).

$F c c=0,515-\mathrm{e}^{(-0,644-(0,515 \mathrm{IAF})}$

em que: IAF é o índice de área foliar, que varia em função da fase fenológica do café. O IAF pode ser estimado pela equação 4 , proposta por Norman et al. (2003).

$\mathrm{IAF}=-2 \ln (1-\mathrm{Fc})$

em que: Fc é a fração do solo coberta pela cultura.

A Fc foi estimada por meio da equação 5, a partir dos valores de índice de vegetação por diferença normalizada (NDVI), conforme Choudhury et al. (1994).

$\mathrm{Fc}=1-\left[(\mathrm{NDIVmáx}-\mathrm{NDIV}) /(\mathrm{NDIVmáx}-\mathrm{NDIV} \text { mín }]^{0,6}(5)\right.$ em que: NDIVmáx e NDIVmín são os valores de máximo e mínimo NDVI da imagem, respectivamente; NDIV é o valor do NDVI de cada pixel da imagem.

O NDVI é uma relação entre medidas espectrais (reflectância - $\rho$ ) de duas bandas, a do infravermelho próximo (700-1.300 nm) e a do vermelho (600-700 $\mathrm{nm})$. Além de reduzir o número de bandas, visa eliminar diferenças sazonais do ângulo solar e minimizar os efeitos da atenuação atmosférica, e pode ser calculado por meio da equação 6 .

$\mathrm{NDVI}=[(\mathrm{IVP}-\mathrm{V}) /(\mathrm{IVP}+\mathrm{V})]$

em que: IVP é a medida da reflectância do alvo na região espectral do infravermelho próximo e $\mathrm{V}$ é a medida da reflectância do alvo na região espectral do vermelho.

Ao introduzir, no modelo agrometeorológico, a componente espectral, expressa pelo NDVI, o modelo passa a ser denominado de agrometeorológico-espectral.

Inicialmente este modelo foi escrito em linguagem Fortran por Rudorff (1985) e mais tarde implementado por Berka et al. (2003) no Sistema para Processamento de Informações Georreferenciadas (Instituto Nacional de Pesquisas Espaciais, 2004), desenvolvido pelo Instituto Nacional de Pesquisas Espaciais (INPE) (Câmara et al., 1996). O modelo visa determinar a penalização da produtividade potencial decorrente da deficiência hídrica, que ocorre pelo somatório da relação entre a evapotranspiração real e a potencial (ETR/ETP) e de ky que relaciona a deficiência hídrica com a demanda de água pela cultura nos estádios fenológicos.

No cálculo do balanço hídrico, são utilizadas as variáveis temperatura média do ar $\left({ }^{\circ} \mathrm{C}\right)$, velocidade do vento a $2 \mathrm{~m}$ de altura $\left(\mathrm{m} \mathrm{dia}^{-1}\right)$, umidade relativa do ar (\%), insolação (horas dia-1) ou radiação solar (cal $\left.\mathrm{cm}^{-2} \mathrm{dia}^{-1}\right)$ e precipitação pluvial $\left(\mathrm{mm} \mathrm{dia}^{-1}\right)$. Esses dados foram obtidos a partir do modelo regional ETA (Chan, 1996), em escala diária, no período de 2003 a 2008. Outros dados necessários ao modelo para o cálculo do balanço hídrico foram: altitude, capacidade de armazenamento de água disponível no solo (CAD) e profundidade do sistema radicular (D).

A altitude foi obtida do modelo digital de elevação do terreno - Shuttle Radar Topography 
Mission-SRTM - disponibilizado na internet pelo United States Geological Survey (USGS). A CAD foi estimada com base em um mapa de classificação de solos, na escala de 1:100.000, cuja digitalização encontra-se disponível em Geominas (1996), em que os valores de CAD (50, 70 ou $100 \mathrm{~mm}$ ) foram atribuídos para cada classe pedológica, de acordo com Rizzi (2004). A profundidade do sistema radicular (D) foi definida como sendo de $1 \mathrm{~m}$ com base no trabalho de Franco \& Inforzato (1946).

O valor do coeficiente da cultura $(\mathrm{kc})$ utilizado neste trabalho foi igual à unidade (1), considerando que o balanço hídrico foi calculado para o cafeeiro em idade adulta (Camargo \& Pereira, 1994). Os valores de ky para a cultura do café foram determinados por Santos \& Camargo (2006) em propriedades rurais no estado de São Paulo.

O IAF do café em cada pixel foi obtido com imagens adquiridas pelo sensor MODIS que, segundo Rizzi et al. (2006a), apresentam potencial para serem utilizadas na estimativa da produtividade em escalas regionais.

No estudo realizado por Rizzi (2004) e Rizzi et al. (2006b) com a cultura da soja, os autores observaram que, no período de máximo desenvolvimento da cultura, o produto MOD15A2 apresentou valores inválidos do índice de área foliar (IAF). Por essa razão, Rizzi et al. (2006a) recomendam que os valores do índice de área foliar (IAF) sejam obtidos com imagens NDVI, contidas no produto MOD13Q1. Assim, optouse por estimar o IAF de cafezais a partir do NDVI (produto MOD13Q1), utilizando imagens referentes a três anos de alta produtividade $(2003 / 2004,2005 / 2006$ e 2007/2008) e dois anos de baixa produtividade (2004/2005 e 2006/2007).

Outro parâmetro requerido pelo modelo agrometeorológico-espectral é o Fpa. Esse parâmetro está relacionado com a quantidade de massa de matéria seca da cultura que é contabilizada como rendimento. No caso da planta de café, esse parâmetro se refere ao quanto de massa de matéria seca é contabilizado no grão. Em virtude da bienalidade do cafeeiro, foi preciso determinar um valor do Fpa para um ano de baixa e um de alta produtividade. Neste caso, tomou-se como referência os anos-agrícolas 2003/2004, de baixa produtividade, e 2004/2005, de alta produtividade.
Os valores determinados foram empregados no modelo para estimar a produtividade nos demais anos, 2005/2006, 2006/2007 e 2007/2008, respectivamente.

Não existe um valor de Fpa para a cultura do café e, na tentativa de determinar esse valor, tomouse como base valores intermediários do intervalo do Fpa da cultura do algodão $(0,08$ a 0,12$)$, que também produz sementes (Doorenbos \& Kassam, 1979). Para definir o melhor valor de Fpa, as estimativas de produtividade foram confrontadas com as do Instituto Brasileiro de Geografia e Estatística (2010) e selecionaram-se os Fpa correspondentes às estimativas mais próximas.

O valor da PMB $\left(\mathrm{kg} \mathrm{ha}^{-1} \mathrm{dia}^{-1}\right)$ refere-se ao total de massa de matéria seca produzida pela cultura em um dia, adotado com base em Doorenbos \& Kassam (1979). O cálculo desse índice leva em consideração a taxa de produção de uma cultura padrão (20 kg ha-1 por hora), que depende do grupo da cultura $\left(\mathrm{C}_{3}\right.$ ou $\left.\mathrm{C}_{4}\right)$ e da temperatura. Quanto a culturas perenes, o Boletim da FAO (Doorenbos \& Kassam, 1979) apresenta apenas taxa de produção para citros, classificada como cultura de verão com taxa de produção inferior a $20 \mathrm{~kg} \mathrm{ha}^{-1}$ por hora. Não há informação sobre a cultura do café e, tanto pela semelhança da prática de manejo usada na cultura de citros quanto por pertencer ao mesmo grupo de cultura, o $\mathrm{C} 3$, foram adotados de modo empírico, os valores tabelados de citros para o café, o que equivale a uma produção de massa de matéria seca de até $20 \mathrm{~kg} \mathrm{ha}^{-1}$ por hora.

Com a finalidade de obter os valores do NDVI somente em lavouras de café e, posteriormente, calcular o Fc e o IAF (equações 5 e 4), utilizaramse mapas temáticos com a distribuição espacial dos cafezais, obtidos com a interpretação de imagens TM/Landsat-5 (Moreira et al., 2007).

Os dados de produtividade e de produção estimados pelo modelo, por município e por ano-agrícola, foram comparados com os dados estimados pelo IBGE por meio do teste de Kolmogorov-Smirnov. Segundo Marsaglia et al., (2003) quando essa distância entre os dados for maior que o limiar estabelecido pelo nível de probabilidade $(\alpha)$, os conjuntos de dados são considerados estatisticamente diferentes. 


\section{Resultados e Discussão}

O valor de Fpa que melhor ajustou a produtividade em comparação com os valores indicados pelo IBGE foi de 0,048 (Tabela 1). Neste caso, a diferença relativa foi de apenas $0,3 \%$. Ao utilizar esse mesmo valor no ano-agrícola de baixa produtividade, 2004/2005, verificou-se que a diferença relativa entre os resultados obtidos pelo modelo e os dados de referência foi muito grande (41\%). Considerando que o valor de Fpa é um fator multiplicativo no cálculo da produtividade e que esta deveria ser menor do que o resultado obtido pela simulação, decidiu-se testar o modelo com um Fpa menor que 0,048, de maneira empírica. O valor do Fpa igual a 0,032 foi o que melhor se ajustou para esse ano de baixa, com uma diferença relativa de $-3 \%$. Assim, nos anos subsequentes, foi estabelecido um valor $(0,032)$ de Fpa para o ano de alta e outro $(0,048)$ para o ano de baixa produtividade do café.

As estimativas de produtividade, obtidas pelo modelo agrometeorológico-espectral, foram muito próximas às oficiais fornecidas pelo IBGE que, neste trabalho, foram consideradas como um dado de referência, mesmo sendo subjetivas (Tabela 2). De modo geral, a produtividade obtida pelo modelo foi subestimada, em comparação aos dados oficiais. Nos quatros primeiros anos agrícolas, a diferença relativa, em valores absolutos, foi igual ou menor que 5,3\% e, no ano-agrícola 2007/2008, a diferença foi de $-8,5 \%$. Esse resultado pode ser explicado pelo deficit hídrico, que ocorreu no período da floração das lavouras no ano de 2007, e

Tabela 1. Ajuste do fator de produtividade agrícola (Fpa), para os anos de alta produtividade agrícola (2003/2004) e baixa produtividade agrícola $(2004 / 2005)$ de cafezais da mesorregião sul e sudoeste de Minas Gerais ${ }^{(1)}$.

\begin{tabular}{lccc}
\hline Fpa & Pr Modelo $\left(\mathrm{kg} \mathrm{ha}^{-1}\right)$ & Pr IBGE $\left(\mathrm{kg} \mathrm{ha}^{-1}\right)$ & DR $(\%)$ \\
\hline & & $2003 / 2004$ \\
0,046 & 1.125 & 1.175 & $-4,3$ \\
0,048 & 1.171 & 1.175 & $-0,3$ \\
0,050 & 1.224 & 1.175 & 4,2 \\
\hline & & $2004 / 2005$ & \\
0,048 & 1.160 & 823 & 41,0 \\
0,035 & 846 & 823 & 2,8 \\
0,032 & 798 & 823 & $-3,0$ \\
\hline
\end{tabular}

(1)Pr, produtividade; DR, diferença relativa da estimativa em relação a referência. pode ser observado nos resultados espacializados da produtividade final (Figura 1). Dessa forma, nota-se que o modelo é bastante sensível a essas variações climáticas (Rizzi \& Rudorff, 2007).

Pôde-se, também, perceber a alternância nos valores estimados entre os anos em estudo, o que condiz com a bienalidade produtiva da cultura. Maiores valores foram obtidos em anos de alta produtividade, que indicam registros de $1.171 \mathrm{~kg} \mathrm{ha}^{-1}$, em 2003/2004, $1.238 \mathrm{~kg} \mathrm{ha}^{-1}$, em 2005/2006 e $1.040 \mathrm{~kg} \mathrm{ha}^{-1}$, em 2007/2008; e menores valores para os anos de baixa produtividade, com registros de $798 \mathrm{~kg} \mathrm{ha}^{-1}$, em 2004/2005 e $805 \mathrm{~kg} \mathrm{ha}^{-1}$, em 2006/2007.

Por meio desse modelo foi possível monitorar, de forma espacializada, a penalização da cultura por período fenológico (Figura 2). Assim, áreas mais atingidas podem ser visitadas e quantificadas em campo, de forma a fornecer informações sobre as condições de cultivo, que podem ser introduzidas no modelo pelos novos parâmetros, na busca de melhorar a exatidão das estimativas.

Independentemente do ano-agrícola e da bienalidade, nota-se que o café foi muito sensível aos fatores climáticos na fase de florada e expansão dos frutos. Nessa fase fenológica, a penalização sobre a produtividade final do café, em termos percentuais, foi de 20,$7 ; 14,4,17,3 ; 14,4$ e 30,2 , respectivamente, para os anos de 2003/2004, 2004/2005, 2005/2006, 2006/2007 e 2007/2008 (Figura 2). A penalização sobre a produtividade do café, nessa fase fenológica, foi mais drástica nos anos-agrícolas 2003/2004, 2004/2005 e 2006/2007, comparado aos outros dois anos. Além de poder estimar a produtividade e a penalização várias vezes ao longo do ciclo fenológico da cultura, essa abordagem permite espacializar os resultados no âmbito municipal.

Tabela 2. Produtividade estimada pelo modelo agrometeorológico-espectral de cafezais da mesorregião sul e sudoeste de Minas Gerais.

\begin{tabular}{lccccc}
\hline Ano-agrícola & \multicolumn{2}{c}{ Produtividade $\left(\mathrm{kg} \mathrm{ha}^{-1}\right)$} & & \multicolumn{2}{c}{ Diferença } \\
\cline { 2 - 3 } \cline { 5 - 6 } & Modelo & IBGE & & $\left(\mathrm{kg} \mathrm{ha}^{-1}\right)$ & $(\%)$ \\
\hline $2003 / 2004$ & 1.171 & 1.175 & & 4 & $-0,4$ \\
$2004 / 2005$ & 798 & 823 & & 5 & $-3,0$ \\
$2005 / 2006$ & 1.238 & 1.308 & & 26 & $-5,3$ \\
$2006 / 2007$ & 805 & 817 & & 12 & $-1,5$ \\
$2007 / 2008$ & 1.137 & 1.040 & & 97 & $-8,5$ \\
\hline
\end{tabular}


As produtividades parciais médias foram acumuladas ao longo do ciclo fenológico da cultura e plotadas em um gráfico (Figura 3). Mais de $80 \%$ da produtividade final foi estimada até a primeira quinzena do mês de fevereiro para todos os cinco anos-agrícolas analisados.

As produtividades do café, obtidas por meio do modelo e pelo IBGE, para cada município da área de estudo, nos cinco anos-agrícolas estão apresentadas na Figura 4. Nota-se que, independentemente do ano-agrícola, a produtividade divulgada pelo IBGE por município oscilou muito mais do que a estimada pelo modelo. Nos anos de alta produtividade - 2003/2004,
2005/2006 e 2007/2008 - a produtividade do café fornecida pelo IBGE variou de 330 a $2.400 \mathrm{~kg} \mathrm{ha}^{-1}$. A produtividade estimada pelo modelo apresentou um intervalo de variação de 620 a $1.400 \mathrm{~kg} \mathrm{ha}^{-1}$. Nos anos de baixa produtividade (2004/2005 e 2006/2007), a produtividade fornecida pelo IBGE variou de 300 a $1.500 \mathrm{~kg} \mathrm{ha}^{-1}$, enquanto a obtida por meio do modelo variou de 620 a $1.000 \mathrm{~kg} \mathrm{ha}^{-1}$. Essa diferença está relacionada a alguns fatores como variedade, idade das lavouras e diferentes práticas de manejo (adensamento e uso de irrigação), tipo e fertilidade dos solos, além de diferentes métodos de coleta das informações.
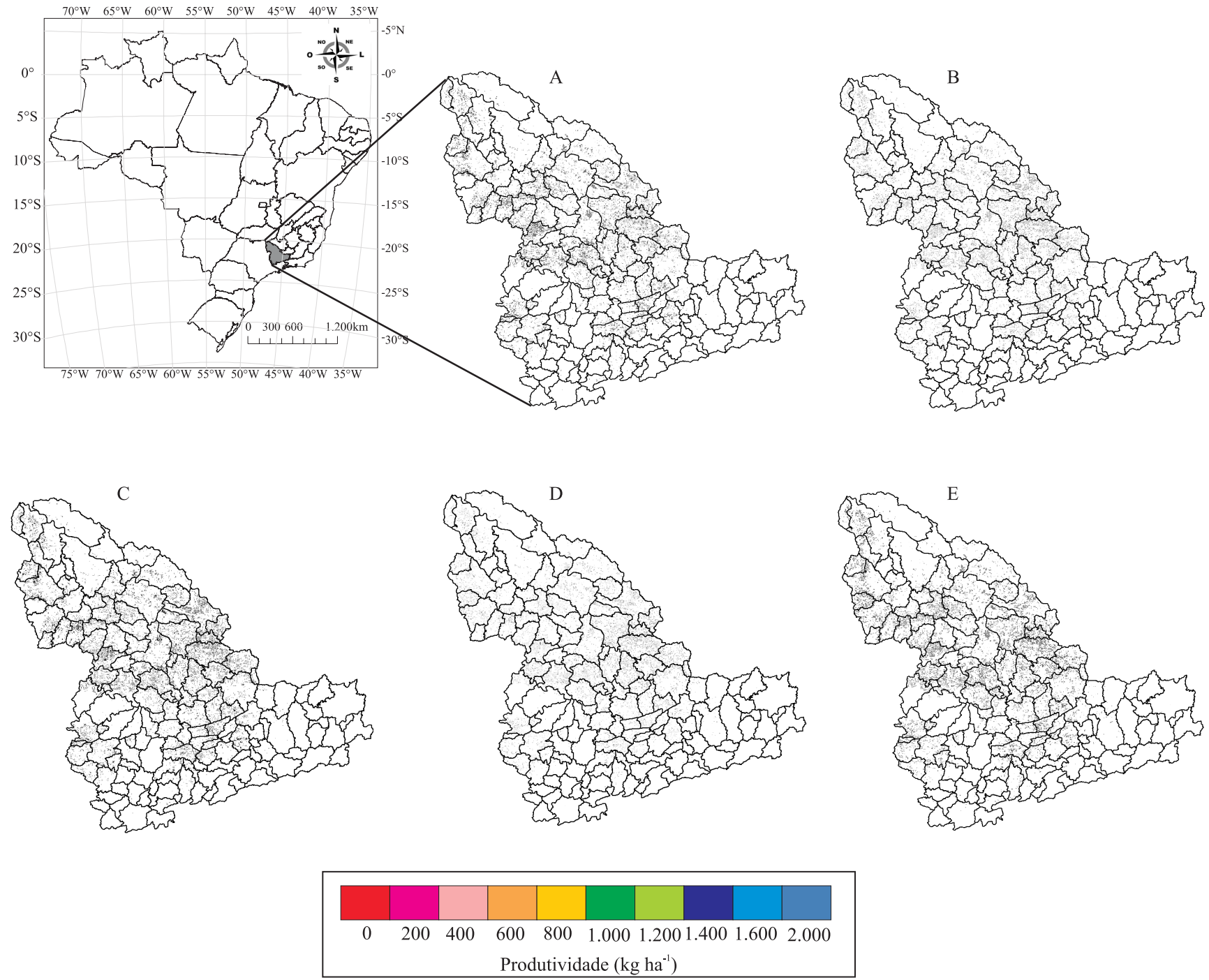

Figura 1. Produtividade média da região estimada nos anos-agrícolas: A, ano 2003/2004 Pr 1.171 kg ha-1; B, 2004/2005 Pr $798 \mathrm{~kg} \mathrm{ha}^{-1}$; C, 2005/2006 Pr 1.231 kg ha ${ }^{-1}$; D, 2006/2007 Pr $805 \mathrm{~kg} \mathrm{ha}^{-1}$; E, 2007/2008 Pr $1.040 \mathrm{~kg}^{-1}$ na mesorregião sul e sudoeste do Estado de Minas Gerais. 

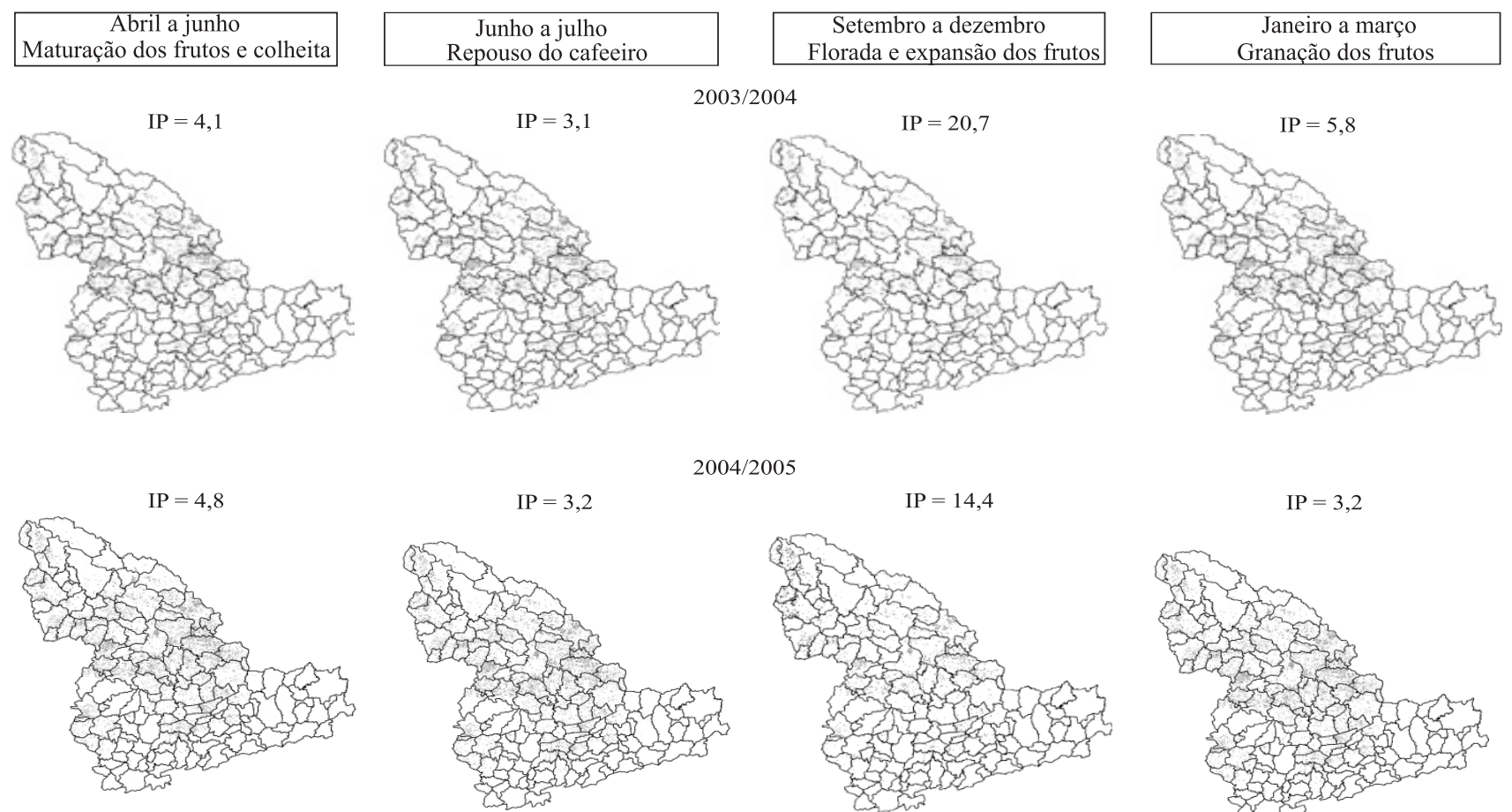

$2004 / 2005$
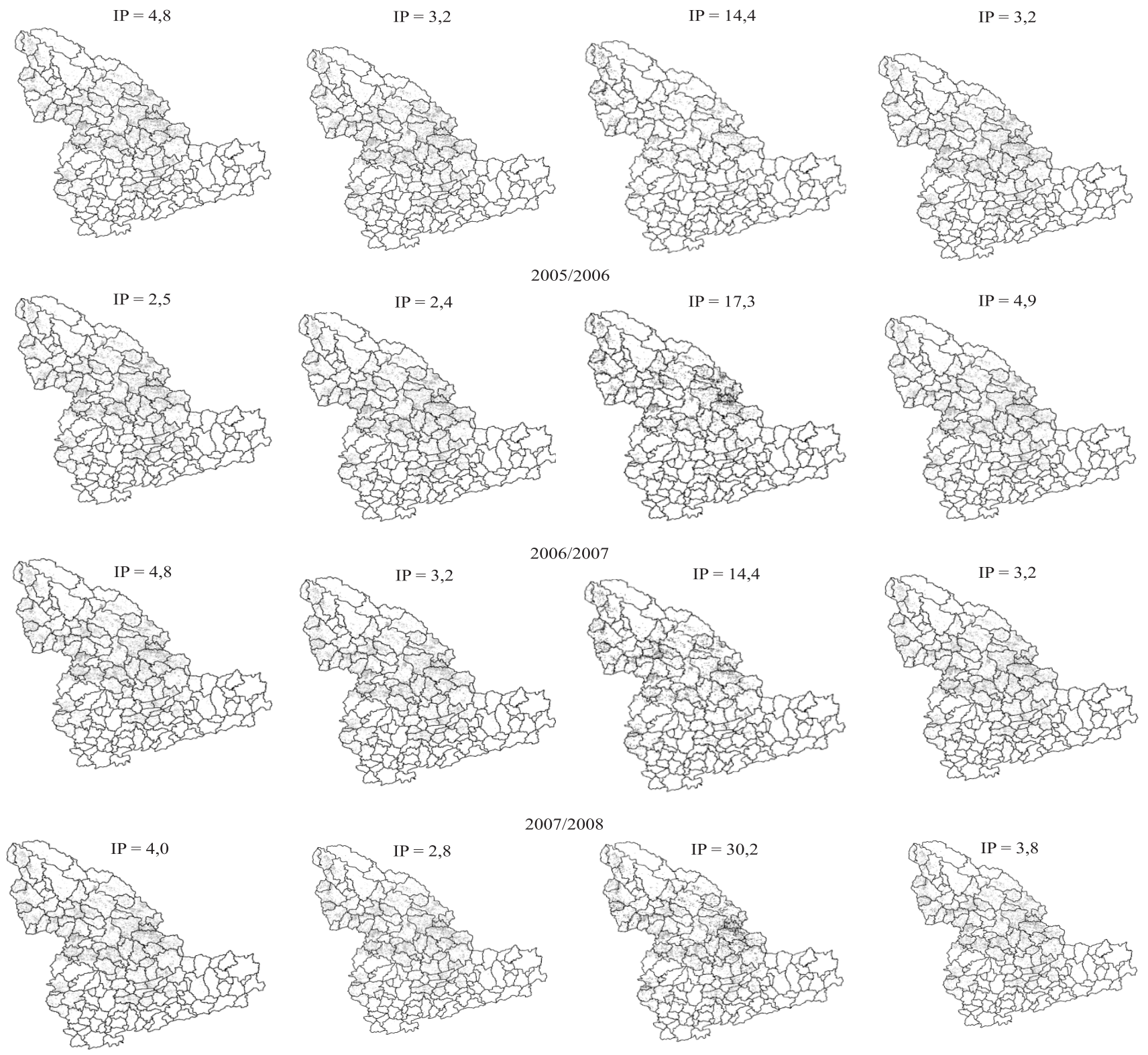

Figura 2. Espacialização do índice de penalização médio acumulado (IP) em percentagem, por período fenológico de cafezais, nos anos-agrícolas 2003/2004, 2004/2005, 2005/2006, 2006/2007 e 2007/2008. 


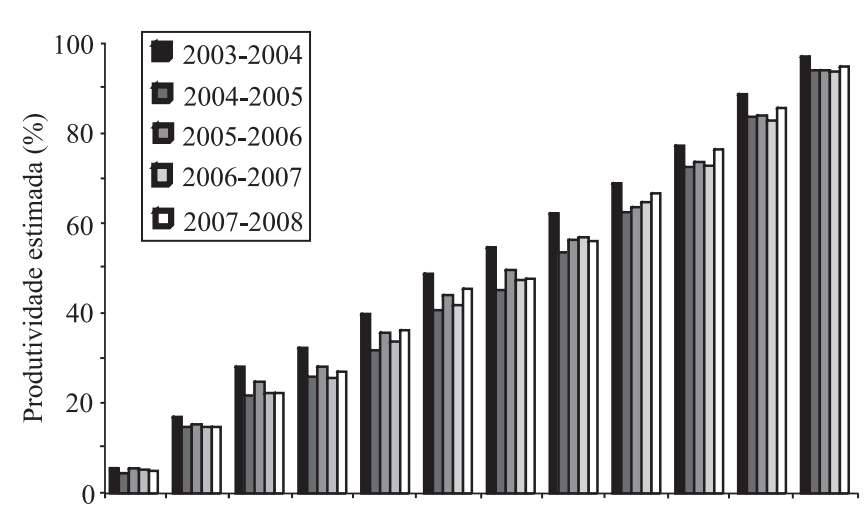

Abr. Maio Jun. Jul. Ago. Set. Out. Nov. Dez. Jan. Fev. Mar.

Meses do ano

Figura 3. Produtividade estimada acumulada durante o ciclo fenológico da cultura do café para os anos-agrícolas 2003/2004, 2004/2005, 2005/2006, 2006/2007 e 2007/2008.
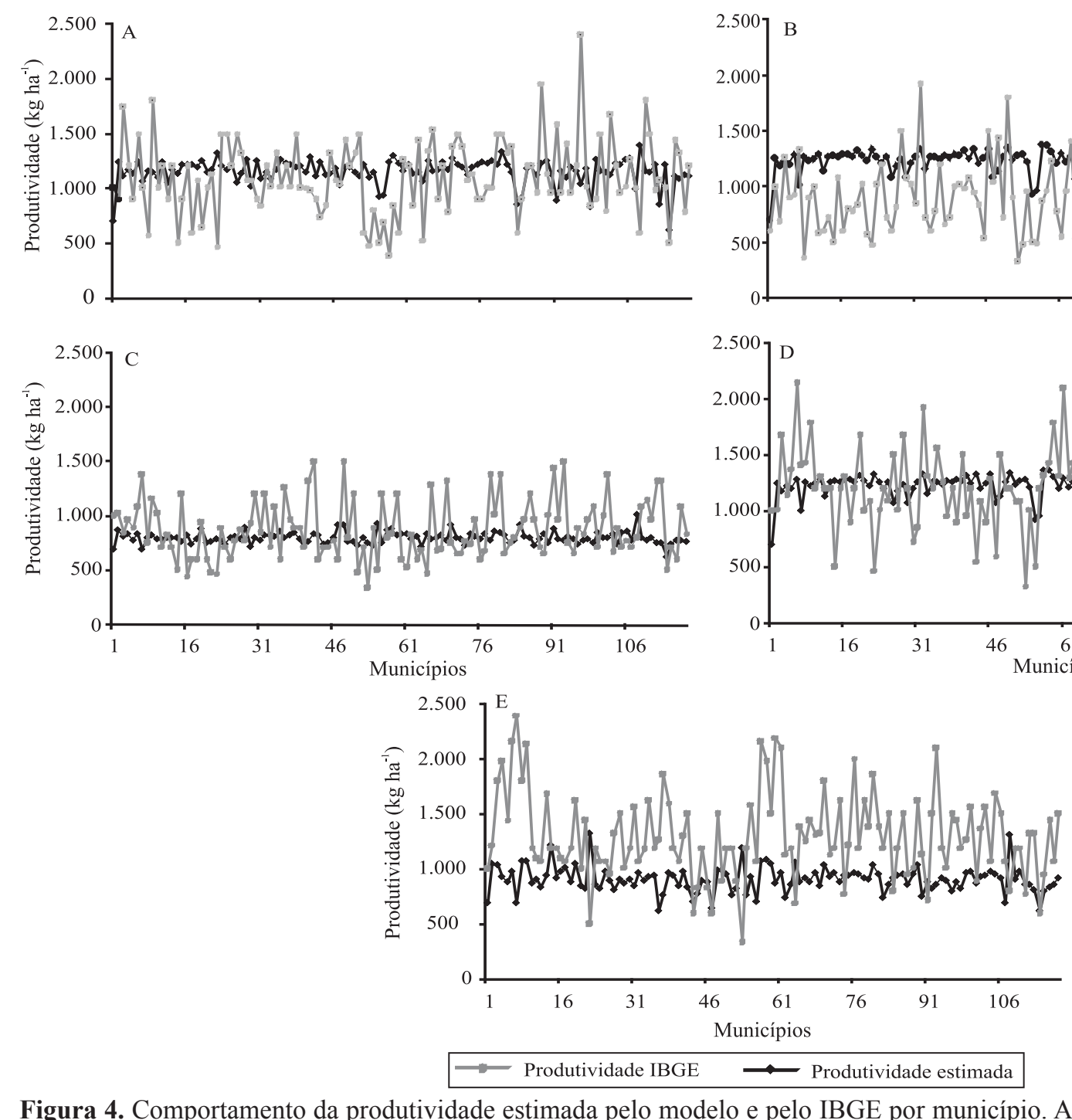

Figura 4. Comportamento da produtividade estimada pelo modelo e pelo IBGE por município. A, ano-agrícola 2003/2004; B, 2004/2005; C, 2005/2006; D, 2006/2007; E, 2007/2008.

Uma das justificativas para essa diferença entre os dois registros é que os valores de NDVI usados na estimativa do IAF são valores médios encontrados em pixels de $250 \times 250 \mathrm{~m}$, o que de certa forma mascara detalhes e diversidades encontradas nesses 6,25 ha de área de café.

Os valores dos coeficientes de determinação $\left(\mathrm{R}^{2}\right)$ para os anos-agrícolas 2003/2004, 2004/2005, 2005/2006 e 2006/2007 foram: 0,76; 0,78; 0,87 e 0,89 , respectivamente (Figura 5). Esse resultado mostra que o modelo explicou 76, 78, 87 e $89 \%$ da produção divulgada pelo IBGE. Ao analisar os resultados das Figuras 4 e 5, observa-se que, no âmbito municipal, a metodologia utilizada pelo IBGE, para determinar a produção de café,
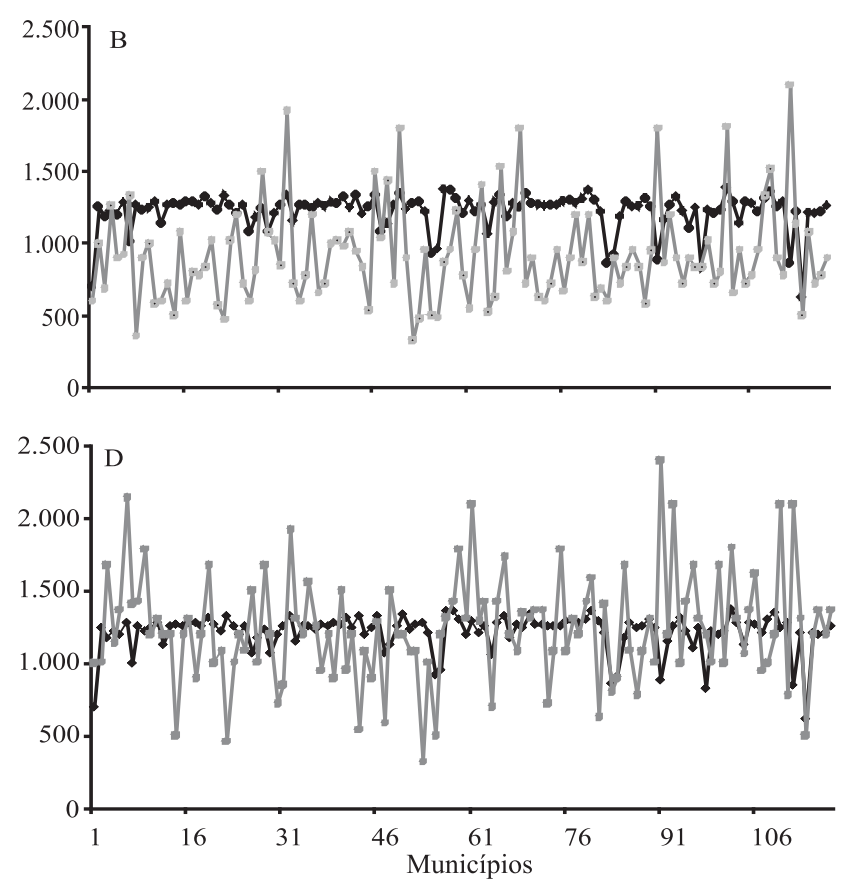
é mais consistente do que a utilizada para o cálculo da produtividade, o que concorda com Rizzi (2004). De acordo com esse autor, o IBGE tem boa estimativa de produção, entretanto, por falta de metodologia objetiva para estimar a área cultivada, os valores de produtividade oficiais do IBGE são discrepantes.

No ano-agrícola 2007/2008, o coeficiente de determinação foi de $\mathrm{R}^{2}=0,54$, ou seja, o modelo explicou apenas $54 \%$ da produção fornecida pelo
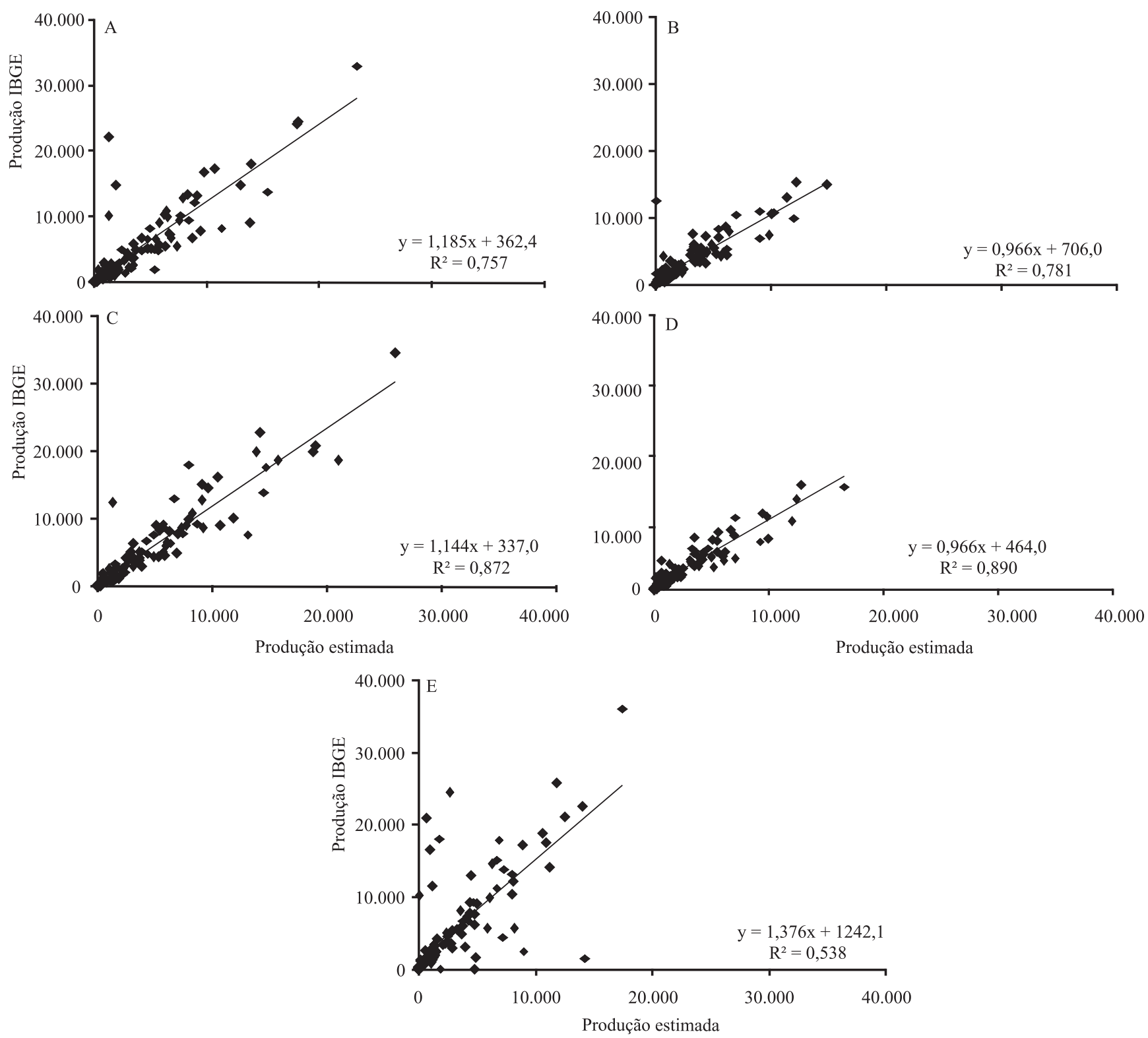

Figura 5. Análise da correlação entre a produção ( $\mathrm{Mg}$ por município) estimada pelo modelo e a do IBGE para os anos-agícolas: A, 2003/2004; B, 2004/2005: C, 2005/2006: D, 2006/2007 e E, 2007/2008. 
período de floração no ano de 2007, conforme pode ser visualizado no balanço hídrico (Figura 6). Esse fato permite inferir que o modelo foi sensível aos efeitos do deficit hídrico e da temperatura, pois os valores de produtividade estimados foram baixos. A explicação mais provável dessa baixa correlação pode ser a defasagem na atualização dos dados de produtividade informados pelos agentes de coleta, e que superestimou a produção na maioria dos municípios.

A produtividade estimada pelo modelo agrometeorológico-espectral teve um comportamento muito similar nos cinco anos-agrícolas estudados (Figura 4), o que demonstra a consistência nos resultados obtidos pelo modelo.

Os resultados do teste Kolmogorov-Smirnov mostram que há diferença significativa entre a produtividade de café estimada pelo modelo e a oficial do IBGE (Tabela 3). No entanto, não

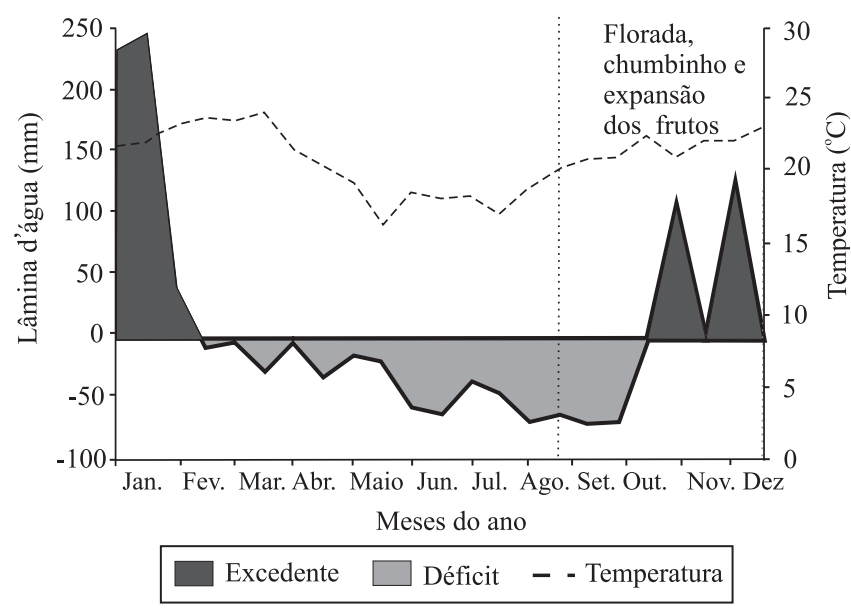

Figura 6. Balanço hídrico da mesorregião sul e sudoeste de Minas Gerais para o ano de 2007.

Tabela 3. Teste de Kolmogorov-Smirnov (KS) para comparar a produtividade e produção de cafezais estimadas pelo modelo com aquelas que foram estimadas pelo IBGE, por município e por ano-agrícola da mesorregião sul e sudoeste de Minas Gerais.

\begin{tabular}{lccccc}
\hline Ano-agrícola & \multicolumn{2}{c}{ Produtividade } & & \multicolumn{2}{c}{ Produção } \\
\cline { 2 - 3 } \cline { 5 - 6 } & $\mathrm{KS}^{(1)}$ & $\mathrm{p}$-value & & $\mathrm{KS}^{(1)}$ & $\mathrm{p}$-value \\
\hline $2003 / 2004$ & 0,41 & 0,00 & & 0,09 & 0,66 \\
$2004 / 2005$ & 0,75 & 0,00 & & 0,11 & 0,45 \\
$2005 / 2006$ & 0,33 & 0,00 & & 0,09 & 0,66 \\
$2006 / 2007$ & 0,39 & 0,00 & & 0,11 & 0,36 \\
$2007 / 2008$ & 0,75 & 0,00 & & 0,18 & 0,03 \\
\hline
\end{tabular}

${ }^{(1)}$ Distância entre os dois conjuntos de dados. se observou diferença significativa $(\alpha=0,01)$ para a produção em nenhum ano-agrícola. Esses resultados condizem com a discussão dos dados contidos nas Figuras 4 e 5. Em todos os anos, observa-se presença de pontos discrepantes, que representam municípios com produção distinta entre a fornecida pelo IBGE e a estimada pelo modelo. Essas inconsistências podem ser resultado da forma utilizada para estimar a área pelo IBGE e pelo mapeamento das lavouras cafeeiras por meio de imagens do TM/Landsat.

\section{Conclusões}

1. O modelo agrometeorológico-espectral, com base nos dados do sensor MODIS a bordo da plataforma Terra e nos dados do modelo regional ETA, é eficaz para estimar a produtividade de cafezais.

2. Pelo modelo agrometeorológico-espectral é possível determinar $80 \%$ da produtividade final até a primeira quinzena do mês de fevereiro, antes de começar a colheita do café.

\section{Referências}

BERKA, L.M.S.; RUDORFF, B.F.T.; SHIMABUKURO, Y.E. Soybean yield estimation by an agrometeorological model in a GIS. Scientia Agricola, v.60, p.433-440, 2003.

CÂMARA G.; SOUZA, R.C.M.; FREITAS, U.M.; GARRIDO, J. SPRING: Integrating remote sensing and GIS by object-oriented data modelling. Computers \& Graphics, v.20, p.395-403, 1996.

CAMARGO, Â.P. de; CAMARGO M.B.P. de. Definição e esquematização das fases fenológicas do cafeeiro arábica nas condições tropicais do Brasil. Bragantia, v.60, p.65-68, 2001.

CAMARGO, Â.P. de; PEREIRA, A.R. Agrometeorology of the coffee crop. Geneva: World Meteorological Organization, 1994. $43 p$.

CAMARGO, M.B.P. de; ORTOLONI, A.A.; PEDRO JÚNIOR, M.J.; ROSA, S.M. Modelo agrometeorológico de estimativa de produtividade para o cultivar de laranja Valência. Bragantia, v.58, p.171-178, 1999.

CARVALHO, L.G.; SEDIYAMA, G.C.; CECON, P.R.; ALVEZ, H.M.R. Avaliação de um modelo agrometeorológico para previsão da produtividade de café em três localidades da região Sul do Estado de Minas Gerais. Revista Brasileira de Agrometeorologia, v.11, p.343-352, 2003.

CHAN, S.C. Modelo regional ETA. Climanálise, v.10, 1996. Edição especial comemorativa de 10 anos. Disponível em: $<$ http:// www6.cptec.inpe.br/products/climanalise/cliesp10a/index1.html>. Acesso em: 7 dez. 2005. 
CHOUDHURY, B.J.; AHAMED, N.U.; IDSO, S.B.; REGINATO, R.J.; DAUGHTRY, C.S.T. Relations between evaporation coefficients and vegetation indices studied by model simulations. Remote Sensing of Environment, v.50, p.1-17, 1994.

DOORENBOS, J.; KASSAM, A.H. Yield response to water. Rome: FAO, 1979. 193p. (FAO. Irrigation and Drainage Paper, 33).

FRANCO, C.M.; INFORZATO, R. O sistema radicular do cafeeiro nos principais tipos de solos do Estado de São Paulo. Bragantia, v.6, p.444-478, 1946.

GEOMINAS. Programa integrado de uso da tecnologia de geoprocessamento pelos órgãos do Estado de Minas Gerais: mapas/downloads. Disponível em: <http://www.geominas.mg.gov. br>. Acesso em: 13 out 2005.

INSTITUTO BRASILEIRO DE GEOGRAFIA E ESTATÍSTICA. Pesquisa Industrial Mensal Produção Física - Regional. Disponível em: <http://www.ibge.gov.br/home/estatistica/ indicadores/industria/pimpfregional/default.shtm>. Acesso em: 06 dez. 2010.

INSTITUTO NACIONAL DE PESQUISAS ESPACIAIS. SPRING: sistema de processamento de informações georeferencias: tutoriais. Versão 4.1. São José dos Campos: INPE, 2004.

LIU, W.T.H.; LIU, B.W.Y. Comparação de três modelos de previsão de safra de café no Estado de Minas Gerais. Ciência e Cultura, v.40, p.801-807, 1988.

MARSAGLIA, G.; TSANG, W.W.; WANG, J. Evaluating Kolmogorov's distribution. Journal of Statistical Software, v.8, p.1-4, 2003.

MOREIRA, M.A.; BARROS, M.A.; FARIA, V.G.C. de;ADAMI, M. Tecnologia de informação: imagens de satélite para o mapeamento de áreas de café de Minas Gerais. Informe Agropecuário, v.28, p.27-37, 2007.

NORMAN, J.M.; ANDERSON, M.C.; KUSTAS, W.P.; FRENCH, A.N.; MECIKALSKI, J.; TORN, R.; DIAK, G.R.; SCHMUGGE, T.J. Remote sensing of evapotranspiration for precision-farming applications. In: INTERNATIONAL GEOSCIENCE AND REMOTE SENSING SYMPOSIUM, 2003, Tolouse. Proceedings. Tolouse: IGARSS, 2003. p.21-25.

PICINI, A.G.; CAMARGO, M.B.P. de; ORTOLANI, A.A.; FAZUOLI, L.C.; GALLO, P.B. Desenvolvimento e teste de modelos agrometeorológicos para a estimativa de produtividade do cafeeiro. Bragantia, v.58, p.157-170, 1999.
PINO, F.A. Estimativa subjetiva de safras agrícolas. Informações Econômicas, v.31, p.55-58, 2001.

RIZZI, R. Geotecnologias em um sistema de estimativa da produção de soja: estudo de caso no Rio Grande do Sul. 2004. 214p. Tese (Doutorado) - Instituto Nacional de Pesquisas Espaciais, São José dos Campos.

RIZZI, R.; RUDORFF, B.F.T. Imagens do sensor MODIS associadas a um modelo agronômico para estimar a produtividade de soja. Pesquisa Agropecuária Brasileira, v.42, p.73-80, 2007.

RIZZI, R.; RUDORFF, B.F.T; FREITAS, R. de; FONTANA, D.C. Monitoramento e estimativa da produtividade de soja auxiliados pelo modelo regional de previsão de tempo ETA. Revista Ambiente e Água, v.1, p.28-38, 2006a.

RIZZI, R.; RUDORFF, B.F.T; SHIMABUKURO, Y.E.; DORAISWAMY, P.C. Assessment of MODIS LAI retrievals over soybean crop in Southern Brazil. International Journal of Remote Sensing, v.27, p.4091-4100, 2006b.

RUDORFF, B.F.T. Dados Landsat na estimativa da produtividade agrícola da cana-de-açúcar. 1985. 114 p. Dissertação (Mestrado) - Instituto Nacional de Pesquisas Espaciais, São José dos Campos.

RUDORFF, B.F.T.; BATISTA, G.T. Yield estimation of sugarcane based on agrometeorological-spectral models. Remote Sensing of Environment, v.33, p.183-192, 1990.

SANTOS, M.A. dos; CAMARGO, M.B.P. de. Parametrização de modelo agrometeorológico de estimativa de produtividade do cafeeiro nas condições do estado de São Paulo. Bragantia, v.65, p.173-183, 2006.

SEDIYAMA, G.C.; MELO JUNIOR, J.C.F. de; SANTOS, A.R. dos; RIBEIRO, A.; COSTA, M.H.; HAMAKAWA, P.J.; COSTA, J.M.N. da; COSTA, L.C. Zoneamento agroclimático do cafeeiro (Coffea arabica L.) para o estado de Minas Gerais. Revista Brasileira de Agrometeorologia, v.9, p.501-509, 2001.

SILVA, G.L.S.P.; VICENTE, J.R.; CASER, D.V. Avaliação de previsões de rendimento de culturas no Estado de São Paulo, fornecidas por modelos agrometeorológicos, anos agrícolas 1984/85 e 1985/86. Informações Econômicas, v.17, p.31-45, 1987.

TOSELLO, R.N.; ARRUDA, H.V. de. Correlação entre estimativas oficiais de produção de café e precipitação pluviométrica, no Estado de São Paulo. Bragantia, v.21, p.449-465, 1962.

WEILL, M.A.M. Avaliação de fatores edafoclimáticos e do manejo na produção de cafeeiros (Coffea arabica L.) na região de Marília e Garça, SP. 1990. 182p. Dissertação (Mestrado) Escola Superior de Agricultura Luiz de Queiroz, Piracicaba.

Recebido em 20 de julho de 2010 e aprovado em 4 de outubro de 2010

Pesq. agropec. bras., Brasília, v.45, n.12, p.1478-1488, dez. 2010 